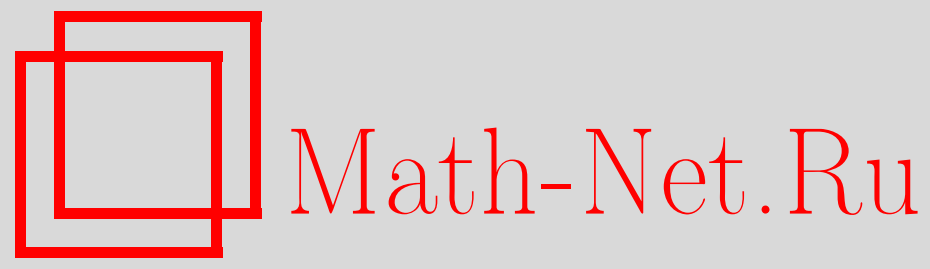

В. П. Радченко, А. Д. Москалик, И. Е. Адеянов, Сравнительный анализ приближенного аналитического и конечно-элементного решений для несоосной трубы, Вестн. Сам. гос. техн. ун-та. Сер. Физ.-мат. науки, 2014, выпуск 3(), 79-93

DOI: https://doi.org/10.14498/vsgtu1340

Использование Общероссийского математического портала MathNet.Ru подразумевает, что вы прочитали и согласны с пользовательским соглашением

http://www.mathnet.ru/rus/agreement

Параметры загрузки:

IP: 54.81 .137 .203

26 апреля 2023 г., 02:16:13

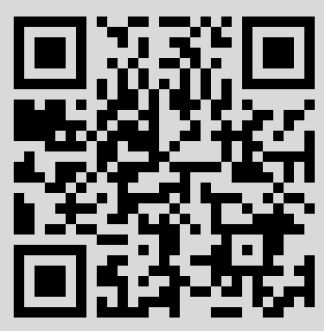




\title{
СРАВНИТЕЛЬНЫЙ АНАЛИЗ ПРИБЛИЖЕННОГО АНАЛИТИЧЕСКОГО И КОНЕЧНО-ЭЛЕМЕНТНОГО РЕШЕНИЙ ДЛЯ НЕСООСНОЙ ТРУБЫ
}

\author{
В. П. Радченко, А. Д. Москалик, И. Е. Адеянов
}

Самарский государственный технический университет,

Россия, 443100, Самара, ул. Молодогвардейская, 244.

\begin{abstract}
Аннотация
Рассмотрена краевая задача установившейся ползучести для несоосной толстостенной трубы, находящейся под внутренним давлением. Строится приближенное аналитическое решение данной задачи методом малого параметра до второго приближения включительно. Решение строится для плоского деформированного состояния. Используется гипотеза несжимаемости материала для деформаций ползучести. В качестве малого параметра используется величина смещения центров внутреннего и внешнего радиусов трубы. Основное внимание уделено вопросу сходимости полученного аналитического решения во втором приближении и оценке его погрешности. Отмечается, что проблема сходимости решена только для краевых задач в упругой области. Поэтому оценка погрешности в поставленной задаче решалась на основании сравнения приближенного аналитического решения с численным решением, построенным методом конечных элементов, для некоторых частных случаев. С учетом симметрии задачи конечно-элементная модель была построена для половины трубы. Количество конечных элементов - около 18000. Вторая половина трубы заменялась граничными условиями с учетом симметрии задачи. Анализ аналитического и численного решений выполнен в зависимости от параметра нелинейности установившейся ползучести и параметра несоосности - отношения смещения центров внешнего и внутреннего диаметров к внешнему радиусу. Показано, что для труб с малыми значениями показателя установившейся ползучести (от 3 до 8) погрешность отклонения приближенного аналитического решения во втором приближении от численного решения вплоть до величины несоосности центров внутреннего и внешнего диаметров 0.1 составляет не более $9 \%$, а для труб с большим показателем нелинейности установившейся ползучести погрешность до $8 \%$ наблюдается при параметре несоосности до 0.06. Приводятся результаты расчетов в табличной форме и в форме графиков. Даны рекомендации по использованию построенного приближенного аналитического решения в прикладных задачах.
\end{abstract}

(C) 2014 Самарский государственный технический университет.

Образец для цитирования: Р ад чен к о В. П., М оск а л и к А. Д., А де ян о в И. Е. Сравнительный анализ приближенного аналитического и конечно-элементного решений для несоосной трубы // Вестн. Сам. гос. техн. ун-та. Сер. Физ.-мат. науки, 2014. № 3 (36). С. 79 93. doi: 10.14498/vsgtu1340.

Сведения об авторах: Владимир Павлович Радченко (д.ф.-м.н., проф.; radch@samgtu.ru), заведующий кафедрой, каф. прикладной математики и информатики. Анна Давидовна Москалик (annmoskalik1@gmail.com; автор, ведущий переписку), аспирант, каф. прикладной математики и информатики. Игорь Евгенъевич Адеянов (к.т.н.; adigorev@gmail.com), доцент, каф. механики. 
Ключевые слова: несоосная толстостенная труба, установившаяся ползучесть, приближенное аналитическое решение, метод малого параметра, второе приближение, конечно-элементная модель, численное решение, погрешность.

doi: http://dx.doi.org/10.14498/vsgtu1340

Введение. Разработка аналитических методов решения краевых задач ползучести для элементов конструкций с возмущенными границами вследствие физической нелинейности определяющих реологических соотношений представляет трудноразрешимую проблему. Один из подходов состоит в линеаризации граничных условий и реологических соотношений на основе метода малого параметра. Число работ в данном направлении необозримо. Однако, что касается внешних краевых задач реологии (с возмущенными границами), то здесь следует отметить ряд работ по устойчивости однородного растяжения полосы или цилиндра из вязкого материала, чувствительного к скорости деформирования, по отношению к малым возмущениям регулярной или произвольной формы свободных границ, решенных методом малого параметpa [1-4]. С другой стороны, развиваются методы решения краевых задач с возмущенным по пространственным переменным полем реологических характеристик (внешние краевые задачи). Так, в работах [5-8] методом малого параметра построены аналитические решения для полей напряжений и скоростей деформаций вплоть до третьего приближения в стохастической краевой задаче установившейся ползучести толстостенной трубы под действием внутреннего давления, а в $[9,10]$ приведены решения аналогичной задачи ползучести для растягиваемой плоскости.

Постановка задачи установившейся ползучести с возмущенными границами методом малого параметра приведена в монографии Л. М. Качанова [11], где, в частности, для несоосной трубы построено решение в первом приближении. Имеются попытки решения этой задачи в работах $[12,13]$.

Детально метод возмущений (малого параметра) для упругопластических тел изложен в монографии [14] и систематически развивался в научной школе Д. Д. Ивлева в работах его учеников [15-18 и др.] для различных условий пластичности, составных упругопластических тел, различных типов концентраторов и т. д.

K сожалению, практически во всех работах по решению внешних и внутренних краевых задач методом малого параметра для сред с реономными свойствами отсутствуют оценки сходимости рядов, поскольку это является сложной и неформализованной задачей. Здесь можно отметить только работу [19], в которой этот вопрос решен для микронеоднородной упругой среды.

В связи с вышеизложенным целью данной работы является сравнение приближенного аналитического решения задачи об установившейся ползучести толстостенной несоосной трубы, находящейся под внутренним давлением, методом малого параметра с учетом приближений до второго включительно и конечно-элементного решения данной задачи с помощью программного комплекса инженерного анализа ANSYS.

1. Постановка задачи. Рассматривается несоосная толстостенная труба с внутренним контуром радиуса $r=a$ и смещенным на малую величину $\delta$ центром внешнего контура радиуса $r=b$ относительно центра внутренней окружности под действием внутреннего давления $q$ (рис. 1). 


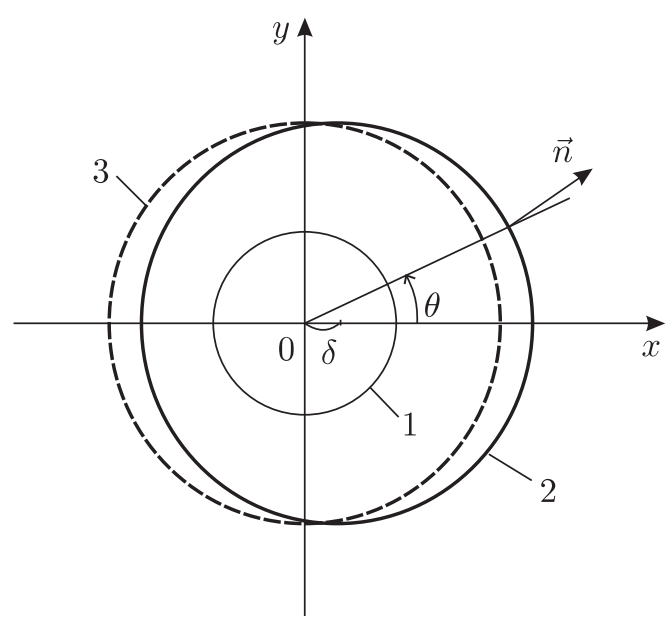

Рис. 1. Схема несоосной трубы: 1 - внутренний контур трубы $r=a ; 2-$ внешний смещенный контур трубы; 3 - внешний контур трубы $r=b$ для осесимметричного случая

[Figure 1. The scheme of a misaligned tube: 1 - the inner contour of the tube; 2 - the outer displaced contour of the tube; 3 - the outer contour of the tube $r=b$ for axisymmetric case;

$\delta$ is the small parameter]

В качестве малого параметра $\delta$ принимается расстояние между центрами внешнего смещенного и внутреннего контуров трубы. При получении приближенного аналитического решения предполагается, что упругие деформации малы по сравнению с деформациями ползучести и ими можно пренебречь. $\mathrm{C}$ физической точки зрения это означает, что рассматриваются установившиеся поля скоростей деформаций ползучести и напряжений, т. е. деформацией ползучести, накопленной на первой стадии и вызванной перераспределением напряжений от упругого состояния до состояния установившейся ползучести, пренебрегаем. Разложение тензора напряжений $\sigma_{i j}$, тензора скоростей деформаций ползучести $\dot{\varepsilon}_{i j}$ и вектора скоростей перемещений $\dot{u}_{i}$ по малому параметру до членов второго порядка имеет вид

$$
\begin{aligned}
& \sigma_{i j}=\sigma_{i j}^{(0)}+\delta \sigma_{i j}^{(1)}+\delta^{2} \sigma_{i j}^{(2)}+\mathrm{O}\left(\delta^{3}\right), \\
& \dot{\varepsilon}_{i j}=\dot{\varepsilon}_{i j}^{0}+\delta \dot{\varepsilon}_{i j}^{(1)}+\delta^{2} \dot{\varepsilon}_{i j}^{(2)}+\mathrm{O}\left(\delta^{3}\right), \\
& \dot{u}_{i}=\dot{u}_{i}^{0}+\delta \dot{u}_{i}^{(1)}+\delta^{2} \dot{u}_{i}^{(2)}+\mathrm{O}\left(\delta^{3}\right),
\end{aligned}
$$

где индексы 0,1 и 2 соответствуют нулевому, первому и второму приближениям.

Уравнение внешнего контура трубы с учетом возмущения $\delta$ имеет вид

$$
(r \cos \theta-\delta)^{2}+r^{2} \sin ^{2} \theta=b^{2} .
$$

Раскладывая последнее соотношение в степенной ряд по параметру $\delta$ и ограничиваясь членами второго порядка включительно, получаем

$$
r=b+\delta \cos \theta+\delta^{2}(\cos 2 \theta-1) / 4 b .
$$

Задача решается в условиях плоского деформированного состояния:

$$
\dot{\varepsilon}_{z z}=0 \text {. }
$$


Предполагается несжимаемость материала для скоростей деформаций ползучести, что находит экспериментальное подтверждение [20,21]:

$$
\dot{\varepsilon}_{r r}+\dot{\varepsilon}_{\theta \theta}=0
$$

Постановка задачи включает в себя уравнения равновесия

$$
\begin{gathered}
\frac{\partial \sigma_{r r}}{\partial r}=-\frac{1}{r} \frac{\partial \sigma_{r \theta}}{\partial \theta}-\frac{\sigma_{r r}-\sigma_{\theta \theta}}{r}, \\
\frac{\partial \sigma_{\theta \theta}}{\partial \theta}=-r \frac{\partial \sigma_{r \theta}}{\partial r}-2 \sigma_{r \theta}
\end{gathered}
$$

которые линейны относительно компонент напряжений и, следовательно, выполняются для каждого приближения.

Аналогично, для каждого приближения выполняются уравнения совместности деформаций

$$
\begin{aligned}
\dot{\varepsilon}_{r r} & =\frac{\partial \dot{u}_{r}}{\partial r}, \\
\dot{\varepsilon}_{\theta \theta} & =\frac{1}{r} \frac{\partial \dot{u}_{\theta}}{\partial \theta}+\frac{\dot{u}_{r}}{r}, \\
\dot{\varepsilon}_{r \theta} & =\frac{1}{2}\left(\frac{1}{r} \frac{\partial \dot{u}_{r}}{\partial \theta}+\frac{\partial \dot{u}_{\theta}}{\partial r}-\frac{\dot{u}_{\theta}}{r}\right) .
\end{aligned}
$$

В качестве определяющих используются соотношения теории установившейся ползучести со степенным законом

$$
\dot{\varepsilon}_{i j}=\frac{3}{2} A \sigma_{e}^{n-1} S_{i j}
$$

где $n, A-$ постоянные характеристики материала, $S_{i j}=\sigma_{i j}-\sigma_{k k} / 3$ - девиатор напряжений, $\sigma_{e}$ - интенсивность напряжений для случая плоской деформации.

2. Приближенное аналитическое решение задачи. Согласно [21] решение для нулевого приближения имеет вид

$$
\begin{aligned}
\sigma_{r r}^{(0)}(r) & =Q\left[1-(b / r)^{p}\right], \\
\sigma_{\theta \theta}^{(0)}(r) & =Q\left[1-(1-p)(b / r)^{p}\right], \\
\sigma_{z z}^{(0)}(r) & =Q\left[1-(1+p)(b / r)^{p}\right],
\end{aligned}
$$

где

$$
Q=\frac{q}{(b / a)^{p}-1}, \quad p=2 / n .
$$

При этом $\sigma_{r \theta}^{(0)}=0$ ввиду симметричности задачи для нулевого приближения.

Поскольку граница при $r=a$ не возмущена и задано давление $q$, линеаризованное граничное условие на внутреннем радиусе трубы для последующих (после нулевого) приближений представимо в виде

$$
\left.\sigma_{r r}^{(k)}\right|_{r=a}=0,\left.\quad \sigma_{r \theta}^{(k)}\right|_{r=a}=0
$$


где $k=1,2$ - номера приближений.

Линеаризованное граничное условие для первого приближения при $r=b$ согласно [14] зависит от нулевого приближения:

$$
\left.\sigma_{r r}^{(1)}\right|_{r=b}=-\frac{d \sigma_{r r}^{(0)}}{d r} \cos \theta,\left.\quad \sigma_{r \theta}^{(1)}\right|_{r=b}=\Delta \sigma^{(0)} \frac{\sin \theta}{b}
$$

где $\Delta \sigma^{(0)}=\sigma_{r r}^{(0)}-\sigma_{\theta \theta}^{(0)}$.

Для первого приближения согласно [22] выполняется

$$
\sigma_{r r}^{(1)}(r, \theta)=\rho_{r r}^{(1)}(r) \cos \theta, \quad \sigma_{\theta \theta}^{(1)}(r, \theta)=\rho_{\theta \theta}^{(1)}(r) \cos \theta, \quad \sigma_{r \theta}^{(1)}=\rho_{r \theta}^{(1)}(r) \sin \theta .
$$

Функции $\rho_{r r}^{(1)}(r), \rho_{\theta \theta}^{(1)}(r), \rho_{r \theta}^{(1)}(r)$ имеют следующий вид:

$\rho_{r r}^{(1)}(r)=\frac{1}{2 L}\left[C_{11}+C_{12} s(s-4 p) r^{-1}+C_{13} \frac{v(v-4 p)}{w+1} r^{-w-1}++C_{14} \frac{w(w-4 p)}{v+1} r^{-v-1}\right]$,

$\rho_{\theta \theta}^{(1)}(r)=\frac{1}{2 L}\left[C_{11}+C_{12} s^{2} r^{-1}+C_{13} \frac{v(v+4 p w)}{w+1} r^{-w-1}++C_{14} \frac{w(w+4 p v)}{v+1} r^{-v-1}\right]$,

$$
\rho_{r \theta}^{(1)}(r)=\frac{1}{2 L}\left[C_{12} s^{2} r^{-1}+C_{13} v^{2} r^{-w-1}+C_{14} w^{2} r^{-v-1}\right],
$$

где

$L=3 A\left(\frac{\sqrt{3}}{n} b^{p} Q\right)^{n-1}, \quad s=p-2, \quad v=\frac{s+\sqrt{s^{2}+16 p}}{2}, \quad w=\frac{s-\sqrt{s^{2}+16 p}}{2}$.

Здесь константы интегрирования $C_{1 i}, i=1,2,3,4$, определяются из граничных условий для первого приближения.

Линеаризованное граничное условие для второго приближения при $r=b$ согласно [14] зависит от нулевого и первого приближений:

$$
\begin{aligned}
\left.\sigma_{r \theta}^{(2)}\right|_{r=b}= & \frac{1}{2}\left[\frac{\Delta \rho^{(1)}}{b}-\frac{d}{d r}\left(\rho_{r \theta}^{(1)}-\frac{\Delta \sigma^{(0)}}{b}\right)\right] \sin 2 \theta \\
\left.\sigma_{r r}^{(2)}\right|_{r=b}=\frac{1}{2}[ & \left.-\frac{d \rho_{r r}^{(1)}}{d r}-\frac{1}{2} \frac{d^{2} \sigma_{r r}^{(0)}}{d r^{2}}-\frac{1}{2 b} \frac{d \sigma_{r r}^{(0)}}{d r}-\frac{\Delta \sigma^{(0)}}{b^{2}}+\frac{2}{b} \rho_{r \theta}^{(1)}\right] \cos 2 \theta+ \\
& +\frac{1}{2}\left[-\frac{d \rho_{r r}^{(1)}}{d r}-\frac{1}{2} \frac{d^{2} \sigma_{r r}^{(0)}}{d r^{2}}+\frac{1}{2 b} \frac{d \sigma_{r r}^{(0)}}{d r}+\frac{\Delta \sigma^{(0)}}{2 b^{2}}-\frac{1}{b} \rho_{r \theta}^{(1)}\right]
\end{aligned}
$$

Здесь $\Delta \rho^{(1)}=\rho_{r r}^{(1)}-\rho_{\theta \theta}^{(1)}$.

Для второго приближения согласно [23] выполняется:

$$
\begin{aligned}
& \sigma_{r r}^{(2)}(r, \theta)=\sigma_{r r}^{R}(R) \cos 2 \theta+\sigma_{r r}^{\psi}(r), \\
& \sigma_{\theta \theta}^{(2)}(r, \theta)=\sigma_{\theta \theta}^{R}(R) \cos 2 \theta+\sigma_{\theta \theta}^{\psi}(r), \\
& \sigma_{r \theta}^{(2)}(r, \theta)=\sigma_{r \theta}^{R}(R) \sin 2 \theta .
\end{aligned}
$$

Решение зависит от функции $R(r)$, имеющей вид

$$
R(r)=\sum_{k=1}^{4}\left[C_{2 k}(r)+p_{k}\right] m_{k}(r)
$$


Здесь $C_{2 k}(r)$ - известные функции радиуса $r, m_{1}=r^{(p+l) / 2} \cos (h \ln r), m_{2}=$ $=r^{(p+l) / 2} \sin (h \ln r), m_{3}=r^{(p-l) / 2} \cos (h \ln r), m_{4}=r^{(p-l) / 2} \sin (h \ln r), l=l(p)$ и $h=h(p)$ - известные значения для конкретного материала; $p_{k}$ - константы интегрирования.

Использование первого и второго приближений метода возмущений позволяет определить напряжения и скорости деформаций ползучести в несоосной трубе. Исследование необходимости построения третьего и более высоких приближений связано с величиной погрешности первых двух приближений, аналитически оценить которую не представляется возможным. Поэтому оценка погрешности приближенного аналитического решения была выполнена на основании его сравнения с численным решением, построенным на основе метода конечных элементов.

3. Анализ приближенного аналитического решения. В качестве модельного примера рассмотрена труба с внутренним радиусом $a=0.115$ м, внешним радиусом $b=0.15$ м под действием внутреннего давления $q=22.07$ МПа. В качестве материалов рассмотрены углеродистая сталь [24] и жаропрочный сплав ХН73МБТЮ (ЭИ698) [25], реологические характеристики которых следующие:

$\begin{array}{lll}\text { углеродистая сталь: } & n=3.03, & A=9.04 \cdot 10^{-9} \\ \text { ХН73МБТЮ (ЭИ698): } & n=10.96, & A=4.57 \cdot 10^{-33} .\end{array}$

В табл. 1 приведены безразмерные значения тангенциального напряжения на внешней границе трубы в первом $\sigma_{\theta \theta}^{*}(\widetilde{r}, \theta)=\sigma_{\theta \theta}^{(0+1)}(\widetilde{r}, \theta) / \sigma_{\theta \theta}^{(0)}(\widetilde{r}, \theta)$ при $\widetilde{r}=b / a+\widetilde{\delta} \cos \theta, \theta=\pi$ и во втором $\sigma_{\theta \theta}^{* *}(\widetilde{r}, \theta)=\sigma_{\theta \theta}^{(0+1+2)}(\widetilde{r}, \theta) / \sigma_{\theta \theta}^{(0)}(\widetilde{r}, \theta)$ при $\widetilde{r}=b / a+\widetilde{\delta} \cos \theta+\widetilde{\delta}^{2}(\cos 2 \theta-1) / 4 b, \theta=\pi$ приближениях в зависимости от безразмерной величины малого параметра $\widetilde{\delta}=\delta / a$ с шагом 0.01 для углеродистой стали и жаропрочного сплава ХН73МБТЮ (ЭИ698).

Из данных, приведенных в табл. 1, можно сделать вывод, что решение задачи о несоосной трубе имеет тенденцию к сходимости. Однако для теоретического вывода о скорости сходимости решения, построенного по методу малого параметра, данных о первых двух приближениях, вообще говоря, недостаточно. Поэтому следующим шагом исследования является численное решение поставленной задачи и сравнение данных приближенного аналитического и численного решений.

Таблица 1

Значения тангенциального напряжения для несоосной трубы [Values of tangential stresses for misaligned tube]

\begin{tabular}{c|c|c|c|c|c|c|c|c|c|c|c}
\hline$\widetilde{\delta}$ & 0 & 0.01 & 0.02 & 0.03 & 0.04 & 0.05 & 0.06 & 0.07 & 0.08 & 0.09 & 0.1 \\
\hline \multicolumn{10}{c}{ Углеродистая сталь [Carbon Steel] } \\
\hline$\sigma_{\theta \theta}^{*}$ & 1.0 & 1.04 & 1.09 & 1.13 & 1.17 & 1.21 & 1.25 & 1.29 & 1.33 & 1.37 & 1.40 \\
$\sigma_{\theta \theta}^{* *}$ & 1.0 & 1.04 & 1.09 & 1.14 & 1.19 & 1.24 & 1.29 & 1.35 & 1.40 & 1.46 & 1.53 \\
\hline \multicolumn{10}{c}{ ХН73МБТЮ (ЭИ698) [KHN73MBТYU (ÉI698) Alloy] } \\
\hline$\sigma_{\theta \theta}^{*}$ & 1.0 & 1.04 & 1.09 & 1.13 & 1.17 & 1.22 & 1.26 & 1.30 & 1.34 & 1.38 & 1.42 \\
$\sigma_{\theta \theta}^{* *}$ & 1.0 & 1.04 & 1.10 & 1.14 & 1.19 & 1.24 & 1.29 & 1.34 & 1.40 & 1.46 & 1.52 \\
\hline
\end{tabular}


4. Конечно-элементная дискретная модель. Для построения численного решения разработана конечно-элементная модель толстостенной несоосной трубы, находящейся под внутренним давлением, с помощью программного комплекса ANSYS.

Решение выполняется двумя шагами:

1) упругое решение;

2) решение с учётом свойств ползучести материала за время $1 \cdot 10^{3}$ ч, поскольку за это время напряженное состояние выходит на стационарный режим, соответствующий стадии установившейся ползучести.

Модуль упругости $E$ и плотность материала $\rho$ принимаются постоянными для выбранного материала и температуры:

углеродистая сталь: $\quad E=1.56 \cdot 10^{5} \mathrm{MПа,} \rho=7630 \mathrm{kг} / \mathrm{M}^{3}, \quad T=649^{\circ} \mathrm{C}$; ХН73МБТЮ (ЭИ698): $\quad E=1.44 \cdot 10^{5} \mathrm{MПа,} \rho=7900 \mathrm{kг} / \mathrm{m}^{3}, \quad T=775^{\circ} \mathrm{C}$.

В расчетах использовался плоский восьмиузловой элемент PLANE183, пригодный для моделирования плоского деформированного состояния и позволяющий учитывать большие деформации при установившейся ползучести. В силу симметрии задачи конечно-элементная модель строилась для половины несоосной трубы. Количество элементов для половины трубы составляет порядка 18000 . Отсутствующая часть трубы заменена условием симметричности по оси $0 x$ (см. рис. 1).

Для оценки конечно-элементной модели на предварительном этапе решалась задача для осесимметричной трубы, находящейся в условиях установившейся ползучести под внутреннем давлением $q$. Решение задачи в такой постановке соответствует нулевому приближению (1) поставленной задачи, аналитическое решение которой хорошо известно [21].

При значениях времени $t=10$ часов изменения напряжений становятся пренебрежимо малы, а деформации ползучести изменяются линейно, что позволяет говорить о состоянии установившейся ползучести материала при $t \geqslant 10$ часов. В качестве решения задачи в условиях установившейся ползучести использовались данные, соответствующие времени $10^{3}$ часов.

5. Сравнение результатов приближенного аналитического и конечно-элементного решений. В работе проведена оценка погрешности приближенного аналитического и конечно-элементного решений задачи для осесимметричной и несоосной трубы с учетом второго приближения включительно на основе значений радиальных $\sigma_{r r}$ и тангенциальных $\sigma_{\theta \theta}$ напряжений в 15 равноотстоящих точках по координате $r_{i}$ :

$$
a \leqslant r_{i} \leqslant b+\delta \cos \theta+\delta^{2}(\cos 2 \theta-1) / 4, \quad i=1,2, \ldots, 15
$$

при $\theta=0$ и $\theta=\pi$. Вычисление погрешности проведено по двум нормам:

$$
s=\frac{\sum_{i=1}^{15}\left|\sigma_{\omega \omega_{i}}^{(0+1+2)}-\sigma_{\omega \omega_{i}}^{a n s}\right|}{\sum_{i=1}^{15}\left|\sigma_{\omega \omega_{i}}^{a n s}\right|} \quad \text { и } \quad \sigma=\left(\frac{\sum_{i=1}^{15}\left[\sigma_{\omega \omega_{i}}^{(0+1+2)}-\sigma_{\omega \omega_{i}}^{a n s}\right]^{2}}{\sum_{i=1}^{15}\left[\sigma_{\omega \omega_{i}}^{a n s}\right]^{2}}\right)^{1 / 2}, \quad \omega=r, \theta,
$$

где $\sigma_{\omega \omega_{i}}^{(0+1+2)}=\sigma_{\omega \omega}^{(0+1+2)}\left(r_{i}, \theta\right), \sigma_{\omega \omega_{i}}^{a n s}=\sigma_{\omega \omega}^{a n s}\left(r_{i}, \theta\right)$ - расчетные значения для аналитического (два приближения) и численного решений соответственно. В табл. 2 приведены оценки различия результатов расчетов между приближенным аналитическим методом и конечно-элементным методом в процентах 


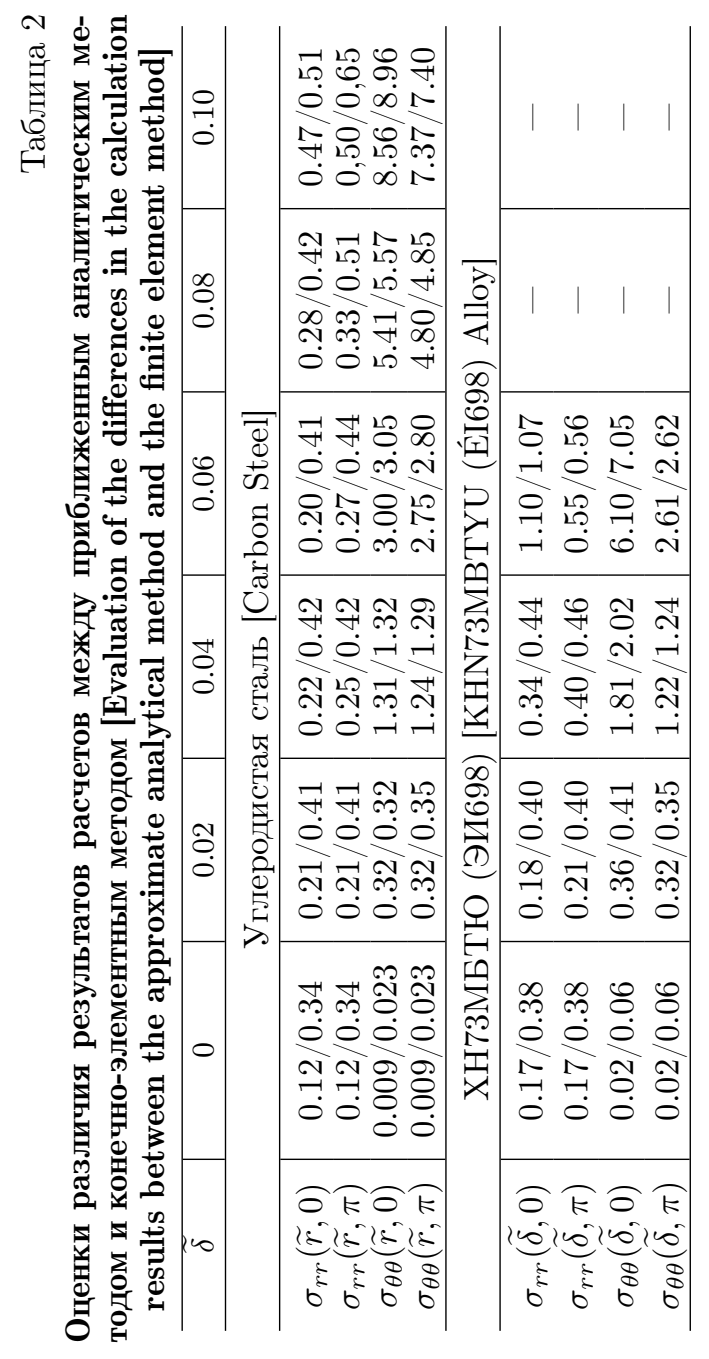



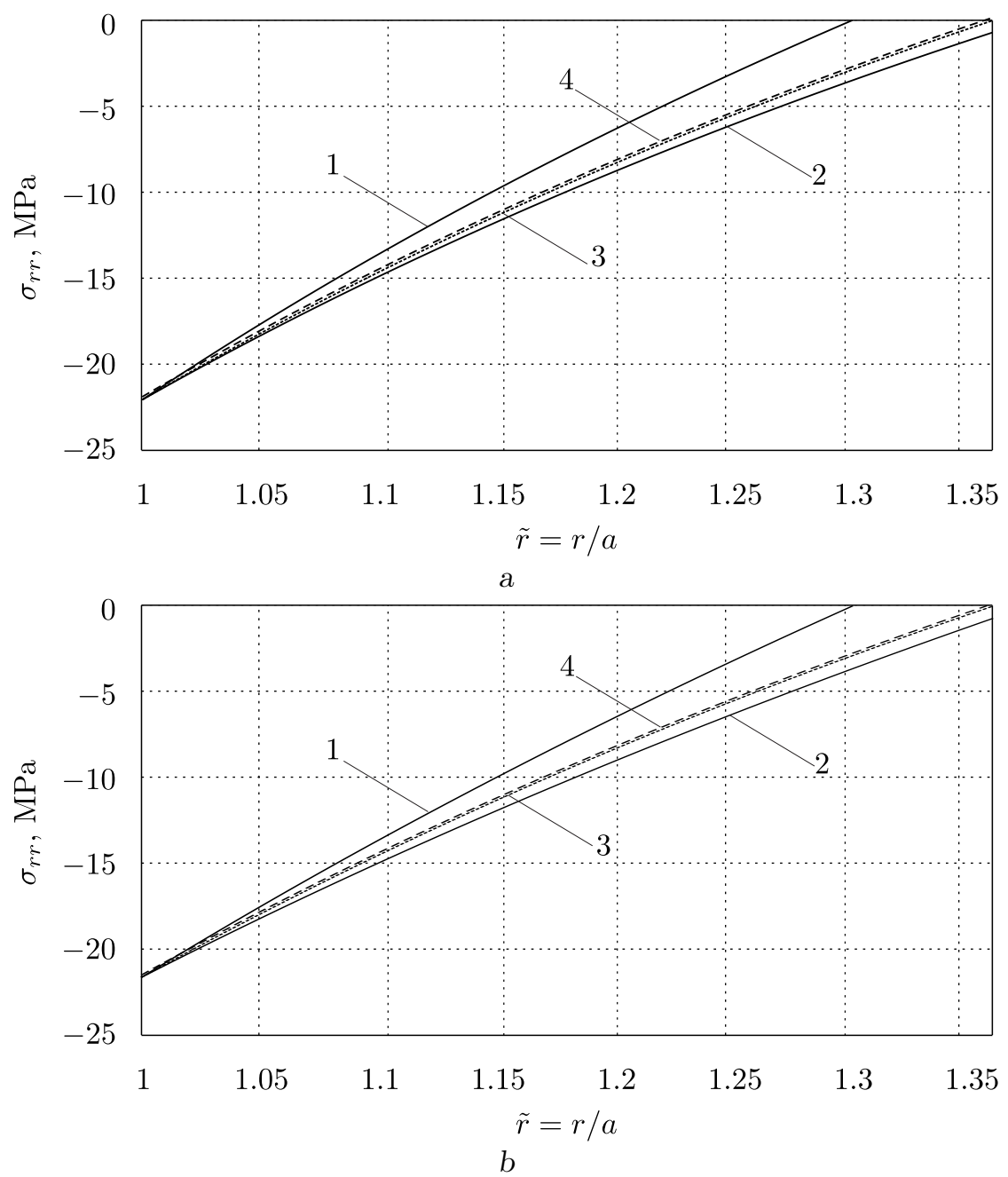

Рис. 2. Радиальные напряжения $\sigma_{r r}$ для несоосных труб из малоуглеродистой стали (а) и сплава ХН73МБТЮ (ЭИ698) (b) при $\theta=0, \tilde{\delta}=0.06: 1-\sigma_{r r}^{(0)}, 2-\sigma_{r r}^{(0+1)}, 3-\sigma_{r r}^{(0+1+2)}, 4-\sigma_{r r}^{\text {Ans }}$

[Figure 2. Radial stresses $\sigma_{r r}$ for carbon steel (a) and KHN73MBTYU (ÉI698) Alloy (b) misaligned tubes, when $\left.\theta=0, \tilde{\delta}=0.06: 1-\sigma_{r r}^{(0)}, 2-\sigma_{r r}^{(0+1)}, 3-\sigma_{r r}^{(0+1+2)}, 4-\sigma_{r r}^{\mathrm{Ans}}\right]$ 

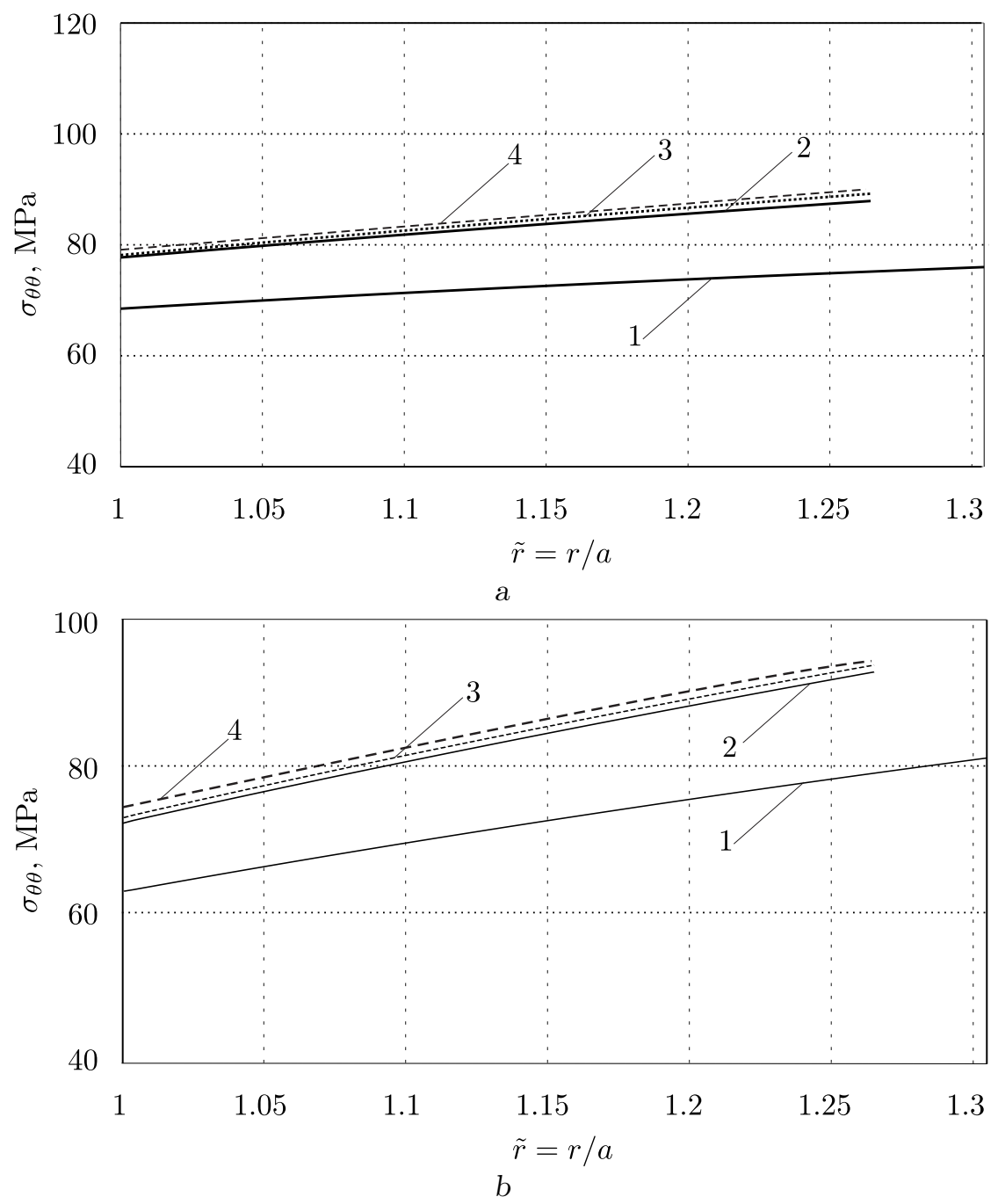

Рис. 3. Тангенциальные напряжения $\sigma_{\theta \theta}$ для несоосных труб из малоуглеродистой стали (а) и сплава ХН73МБТЮ (ЭИ698) (b) при $\theta=\pi, \tilde{\delta}=0.04: 1-\sigma_{\theta \theta}^{(0)}, 2-\sigma_{\theta \theta}^{(0+1)}$, $3-\sigma_{\theta \theta}^{(0+1+2)}, 4-\sigma_{\theta \theta}^{\text {Ans }}$

[Figure 3. Tangential stresses $\sigma_{\theta \theta}$ for carbon steel (a) and KHN73MBTYU (ÉI698) Alloy (b) misaligned tubes, when $\left.\theta=\pi, \tilde{\delta}=0.04: 1-\sigma_{\theta \theta}^{(0)}, 2-\sigma_{\theta \theta}^{(0+1)}, 3-\sigma_{\theta \theta}^{(0+1+2)}, 4-\sigma_{\theta \theta}^{\text {Ans }}\right]$ 
для малоуглеродистой стали и сплава ХН73МБТЮ (ЭИ698), используемых в качестве модельных материалов. Через дробную черту приведены оценки, вычисленные по двум нормам: $s / \sigma$.

В качестве иллюстрации на рис. 2 приведены графики радиальной компоненты $\sigma_{r r}$ тензора напряжений для углеродистой стали $(n=3.03)$ и жаропрочного сплава ХН73МБТЮ (ЭИ698) $(n=10.96)$ при $\theta=0, \tilde{\delta}=0.06$. Верхний индекс у компонент тензора напряжений означает количество приближений, используемых для оценки величин. Индекс (Ans) означает конечно-элементное решение. На рис. 3 приведены графики для тангенциальной компоненты $\sigma_{\theta \theta}$ тензора напряжений при $\tilde{\delta}=0.04$ и значении угла $\theta=\pi$, соответствующего максимальным значениям тангенциального напряжения $\sigma_{\theta \theta}$.

Анализ данных таблицы 2 и графиков на рис. 2, 3 позволяет сделать следующие выводы:

1) для труб с малыми значениями показателя установившейся ползучести материала (малоуглеродистая сталь) погрешность отклонения приближенного аналитического решения во втором приближении от численного решения по методу конечных элементов вплоть до величины несоосности центров внутреннего и внешнего диаметров $\widetilde{\delta}=0.1$ составляет не более $9 \%$; для труб с большим показателем нелинейности установившейся ползучести материала (сплав ХН73МБТЮ (ЭИ698)) для величины $0 \leqslant \widetilde{\delta} \leqslant 0.06$ - погрешность не более $8 \%$, а для значений $\widetilde{\delta}>0.06-$ она существенно возрастает;

2) в расчетной практике для труб с небольшим показателем нелинейности установившейся ползучести материала $(3 \leqslant n \leqslant 8)$ можно ограничиться вторым приближением вплоть до величины $\widetilde{\delta}=0.1$ (с погрешностью решения до $9 \%$ ); для труб с показателем нелинейности установившейся ползучести материала $n>8$ с погрешностью не более $9 \%$ приближенное аналитическое решение во втором приближении можно использовать лишь до величины $\widetilde{\delta}=0.06$, а для величины $\widetilde{\delta}>0.06$, по всей видимости, необходимо иметь приближения более высокого порядка.

\section{ORCID}

Vladimir P. Radchenko: http://orcid.org/0000-0003-4168-9660

Anna Moskalik: http://orcid.org/0000-0001-7527-6237

Igor Adeyanov: http://orcid.org/0000-0002-3943-6173

\section{БИБЛИОГРАФИЧЕСКИЙ СПИСОК}

1. Hill R., Hutchinson J. W. Bifurcation phenomena in the plane tension test // J. Mech. Phys. solids, 1975. vol. 23. pp. 239-264. doi: 10.1016/0022-5096(75)90027-7.

2. Stören S., Rice J. R. Localized necking in thin sheets // J. Mech. Phys. solids, 1975. vol. 23, no. 6. pp. 421-441. doi: 10.1016/0022-5096(75) 90004-6.

3. Hutchinson J. W., Neale K. W. Influence of strain-rate sensitivity on necking under uniaxial tension // Acta Metallurgica, 1977. vol. 25, no. 8. pp. 839-846. doi: 10.1016/0001-6160(77) 90168-7.

4. Келлер И. Э. Равновесные формы свободной границы при одноосном растяжении нелинейно-вязкой полосы // ПМТФ, 2010. Т. 51, № 1. С. 117-124.

5. Радченко В. П., Попов Н. Н. Аналитическое решение стохастической краевой задачи установившейся ползучести для толстостенной трубы // ПММ, 2012. Т. 76, № 6. С. 10231031. 
6. Должковой А. А., Попов Н. Н. Решение нелинейной стохастической задачи ползучести для толстостенной трубы методом малого параметра // Вестн. Сам. гос. техн. ун-та. Сер. Физ.-мат. науки, 2002. №16. С. 84-89. doi: 10.14498/vsgtu102.

7. Попов Н. Н., Исуткина В. Н. Построение аналитического решение двумерной стохастической задачи установившейся ползучести для толстостенной трубы // Becmн. Сам. гос. техн. ун-та. Сер. Физ.-мат. науки, 2007. №2(15). С. 57-61. doi: 10.14498/ vsgtu535.

8. Должковой А. А., Попов Н. Н., Радченко В. П. Решение стохастической краевой задачи установившейся ползучести для толстостенной трубы методом малого параметра // ПМТФ, 2006. Т. 47, № 1. С. 161-171.

9. Коваленко Л. В., Попов Н. Н., Радченко В. П. Решение плоской стохастической краевой задачи ползучести // ПММ, 2009. Т. 73, №6. С. 1009-1016.

10. Попов Н. Н., Самарин Ю. П. Исследование полей напряжений вблизи границы стохастически неоднородной полуплоскости при ползучести // ПМТФ, 1988. № 1. С. 159-164.

11. Качанов Л. М. Теория ползучести. М.: Физматгиз, 1960. 455 с.

12. Радченко В. П., Башкинова Е. В. Решение краевых задач установившейся ползучести в полярных координатах методом возмущений // Вестн. Сам. гос. техн. ун-та. Сер. Техн. науки, 1998. № 5. С. 86-91.

13. Башкинова Е. В. Решение краевой задачи установившейся ползучести для неосесимметричной толстостенной трубы // Вестн. Сам. гос. техн. ун-та. Сер. Физ.-мат. науки, 2002. №16. C. 105-110. doi: 10.14498/vsgtu106.

14. Ивлев Д. Д., Ершов Л. В. Метод возмущений в теории упругопластического тела. М.: Наука, 1978. 208 с.

15. Кержаев А. П. Упругопластическое состояние тонкой кольцевой пластины при наличии трансляционной анизотропии при равномерном растяжении // Вестник Чувашского государственного педагогического университета им. И. Я. Яковлева. Серия: Механика предельного состояния, 2012. № 2(12). С. 174-179.

16. Фоминых С. О. Упругопластическое состояние толстостенной трубы при взаимодействии различных видов пластической анизотропии // Вестник Чувашского государственного педагогического университета им. И. Я. Яковлева. Серия: Механика пределъного состояния, 2011. №1(9). С. 201-2016.

17. Петров Н. И. О деформировании растягиваемой полосы, ослабленной пологими выточками // Вестник Чувашского государственного педагогического университета им. И. Я. Яковлева. Серия: Механика предельного состояния, 2014. № 2(20). С. 36-45.

18. Никитин А. В., Тихонов С. В. Предельное состояние многослойной трансляционноанизотропной толстостенной трубы, находящейся под действием внутреннего давления // Вестник Чувашского государственного педагогического университета им. И. Я. Яковлева. Серия: Механика предельного состояния, 2014. № 1(19). С. 88-94.

19. Кунташев П. А., Немировский Ю. В. О сходимости метода возмущений в задачах теории упругости // Изв. Акад. наук СССР. Мех. тверд. тела, 1985. № 3. С. 75-78.

20. Никитенко А. Ф. Ползучесть и длительная прочность металлических материалов. Новосибирск: НГАСУ, 1997. 278 с.

21. Работнов Ю. Н. Механика деформируемого твердого тела. М.: Наука, 1979. 744 с.

22. Москалик А. Д. Анализ напряженно-деформированного состояния толстостенного несоосного цилиндра, находящегося под внутреннем давлением, в условиях установившейся ползучести методом малого параметра / Труды Девятой Всероссийской научной конференции с международным участием/ Матем. моделирование и краев. задачи. Самара: СамГТУ, 2013. С. 140-144.

23. Москалик А. Д. Применение метода возмущений к задаче о несоосной трубе в условиях установившейся ползучести // Вестн. Сам. гос. техн. ун-та. Сер. Физ.-мат. науки, 2013. № 4(33). С. 76-85. doi: 10.14498/vsgtu1290.

24. Малинин Н. Н. Прикладная теория пластичности и ползучести. М.: Машиностроение, 1975. 400 с. 
25. Радченко В. П., Саушкин М. Н. Ползучесть и релаксация остаточнъх напряжений в упрочненных конструкииях. М.: Машиностроение-1, 2005. 226 с.

Поступила в редакцию 26/V/2014;

в окончательном варианте - 18/VI/2014;

принята в печать - 27/VIII/2014.

Vestn. Samar. Gos. Techn. Un-ta. Ser. Fiz.-mat. nauki. 2014. Issue 3 (36). Pp. $79-93$ [J. Samara State Tech. Univ., Ser. Phys. \& Math. Sci. 2014. Issue 3 (36). Pp. 79-93]

ISSN: 2310-7081 (online), 1991-8615 (print)

doi: http://dx.doi.org/10.14498/vsgtu1340

MSC: 74D10, 74G10

\title{
COMPARATIVE ANALYSIS OF THE APPROXIMATE ANALYTICAL AND FINITE ELEMENT SOLUTIONS FOR MISALIGNED TUBE
}

\author{
V. P. Radchenko, A. D. Moskalik, I. E. Adeyanov
}

Samara State Technical University,

244, Molodogvardeyskaya st., Samara, 443100, Russian Federation.

\begin{abstract}
The boundary value problem of steady-state creep for thick-walled misaligned tube under internal pressure was considered. The approximate analytical solution of this problem by method of small parameter including the second approach is under construction. The solution for the state of plane deformation is constructed. The hypothesis of incompressibility of material for creep strain is used. As a small parameter the misalignment of the centers of the inner and outer radii of the tube is used. The main attention to the convergence of the resulting analytical solution considering the second approximation and assessment of its error is paid. It is noted that the convergence problem is solved only for boundary value problems in the theory of elasticity. Therefore the error assessment in the problem is solved on the basis of a comparison of the approximate analytical solution with the numerical solution constructed on the finite element method, for some special cases. Considering the symmetry of the problem, the finite element model was built for the half tube. The number of finite elements is about 18,000. Considering the symmetry of the problem the second half of the tube is replaced by boundary conditions. Analysis of analytical and numerical solutions is executed depending on the steady-state creep nonlinearity parameter and misaligned parameter that is ratio of the misalignment of the centers of the outer and inner diameter to the outer radius. It is shown that the error
\end{abstract}

(C) 2014 Samara State Technical University.

How to cite Reference: Radchenko V. P., Moskalik A. D., Adeyanov I. E. Comparative Analysis of the Approximate Analytical and Finite Element Solutions for Misaligned Tube, Vestn. Samar. Gos. Tekhn. Univ., Ser. Fiz.-Mat. Nauki [J. Samara State Tech. Univ., Ser. Phys. \& Math. Sci.], 2014, no. 3 (36), pp. 79-93. doi: 10.14498/vsgtu1340. (In Russian)

Authors Details: Vladimir P. Radchenko (Dr. Phys. \& Math. Sci., Professor; radch@samgtu.ru), Head of Department, Dept. of Applied Mathematics \& Computer Science. Anna D. Moskalik (annmoskalik1@gmail.com; Corresponding Author), Postgraduate Student, Dept. of Applied Mathematics \& Computer Science. Igor' E. Adeyanov (Cand. Techn. Sci.; adigorev@gmail.com), Associate Professor, Dept. of Mechanics. 
of deviation of the approximate analytical solution in the second approximation from numerical solution until the misalignment value of the centers of the inner and outer diameters of 0.1 for the tubes with small exponent of the steady-state creep (3 to 8 ) is not more than $9 \%$, and error to $8 \%$ for the tubes with a large exponent of the steady-state creep nonlinearity is observed in the misaligned parameter to 0.06. Results of computations are presented in tabular form and in the form of graphs. Recommendations for the use of the constructed approximate analytical solution in applied problems are given.

Keywords: thick-walled misaligned tube, steady-state creep, approximate analytical solution, small parameter method, second approximation, finite element method, numerical solution, solution error.

doi: http://dx.doi.org/10.14498/vsgtu1340

\section{ORCID}

Vladimir P. Radchenko: http://orcid.org/0000-0003-4168-9660

Anna Moskalik: http://orcid.org/0000-0001-7527-6237

Igor Adeyanov: http://orcid.org/0000-0002-3943-6173

\section{REFERENCES}

1. Hill R., Hutchinson J. W. Bifurcation phenomena in the plane tension test, J. Mech. Phys. solids, 1975, vol. 23, pp. 239-264. doi : 10.1016/0022-5096(75)90027-7.

2. Stören S., Rice J. R. Localized necking in thin sheets, J. Mech. Phys. solids, 1975, vol. 23, no. 6, pp. 421-441. doi: 10.1016/0022-5096(75)90004-6.

3. Hutchinson J. W., Neale K. W. Influence of strain-rate sensitivity on necking under uniaxial tension, Acta Metallurgica, 1977, vol. 25, no. 8, pp. 839-846. doi:10.1016/0001-6160(77) 90168-7.

4. Keller I. É. Self-similar shapes of the free boundary of a nonlinear-viscous band under uniaxial tension, J. Appl. Mech. Tech. Phys., 2010, vol. 51, no. 1, pp. 99-105. doi: 10.1007/ s10808-010-0016-z.

5. Popov N. N., Radchenko V. P. Analytical solution of the stochastic steady-state creep boundary value problem for a thick-walled tube, J. Appl. Math. Mech., 2012, vol.76, no. 6, pp. 738-744. doi: 10.1016/j.jappmathmech.2013.02.011.

6. Dolzhkovoi A. A., Popov N. N. Solution of the nonlinear stochastic creep problem for a thick-walled tube by method of small parameter, Vestn. Samar. Gos. Tekhn. Univ. Ser. Fiz.-Mat. Nauki, 2002, no. 16, pp. 84-89 (In Russian). doi: 10.14498/vsgtu102.

7. Popov N. N., Isutkina V. N. Construction of an Analytical Solution of a Two-Dimensional Stochastic Problem of the Steady Creep for a Thick-Walled Pipe, Vestn. Samar. Gos. Tekhn. Univ. Ser. Fiz.-Mat. Nauki, 2007, no. 2(15), pp. 57-61 (In Russian). doi: 10.14498/ vsgtu535.

8. Dolzhkovoi A. A., Popov N. N., Radchenko V. P. Solution of the stochastic boundaryvalue problem of steady-state creep for a thick-walled tube using the small-parameter method, J. Appl. Mech. Tech. Phys., 2006, vol.47, no.1, pp. 134-142. doi: 10.1007/ s10808-006-0017-0.

9. Kovalenko L. V., Popov N. N., Radchenko V. P. Solution of the plane stochastic creep boundary value problem, J. Appl. Math. Mech., 2009, vol.73, no.6, pp. 727-733. doi: 10 . 1016/j . jappmathmech.2010.01.013.

10. Popov N. N., Samarin Yu. P. Stress fields close to the boundary of a stochastically inhomogeneous half-plane during creep, J. Appl. Mech. Tech. Phys., 1988, vol. 29, no.1, pp. 149-154. doi : 10.1007/BF00909710.

11. Kachanov L. M. Teoriia polzuchesti [Creep theory]. Moscow, Fizmatgiz, 1960, 455 pp. (In Russian) 
12. Radchenko V. P., Bashkinova E. V. Solution of the boundary value problems for steady creep in polar coordinates by the perturbation method, Vestn. Sam. gos. tekhn. un-ta. Ser. Tekhn. nauki, 1998, no. 5, pp. 86-91 (In Russian).

13. Bashkinova E. V. Solution of the value boundary problem of steady creep for nonaxisymmetric thick-walled tube, Vestn. Samar. Gos. Tekhn. Univ. Ser. Fiz.-Mat. Nauki, 16, 2002, pp. 105-110 (In Russian). doi: 10.14498/vsgtu106.

14. Ivlev D. D., Ershov L. V. Metod vozmushchenii $v$ teorii uprugoplasticheskogo tela [Perturbation Method in the Theory of an Elastic-Plastic Body]. M., Nauka, 1978, 208 pp. (In Russian)

15. Kerzhaev A. P. Elastoplastic state of the thin annular plate in the presence of translational anisotropy under uniform tension, Vestnik Chuvashskogo gosudarstvennogo pedagogicheskogo universiteta im. I. Ia. Iakovleva. Seriia: Mekhanika predel'nogo sostoianiia, 2012, no. 2(12), pp. 174-179 (In Russian).

16. Fominykh S. O. Elastoplastic state of the thick-walled pipe by reacting the different types of plastic anisotropy, Vestnik Chuvashskogo gosudarstvennogo pedagogicheskogo universiteta im. I. Ia. Iakovleva. Seriia: Mekhanika predel'nogo sostoianiia, 2011, no. 1(9), pp. 201-2016 (In Russian).

17. Petrov N. I. On the deformation of stretched strip weakened sloping bores, Vestnik Chuvashskogo gosudarstvennogo pedagogicheskogo universiteta im. I. Ia. Iakovleva. Seriia: Mekhanika predel'nogo sostoianiia, 2014, no.2(20), pp. 36-45 (In Russian).

18. Nikitin A. V., Tikhonov S. V. Limit condition anisotropic multilayer translationnally thick-walled pipes under internal pressure, Vestnik Chuvashskogo gosudarstvennogo pedagogicheskogo universiteta im. I. Ia. Iakovleva. Seriia: Mekhanika predel'nogo sostoianiia, 2014, no. 1(19), pp. 88-94 (In Russian).

19. Kuntashev P. A., Nemirovskii Yu. V. Convergence of the perturbation method in elastic problems, Izv. Akad. Nauk SSSR, Mekh. Tverd. Tela, 1985, no. 3, pp. 75-78 (In Russian).

20. Nikitenko A. F. Polzuchest' $i$ dlitel'naia prochnost' metallicheskikh materialov [Creep and Long-Term Strength of Metal Materials]. Novosibirsk, Novosibirsk State University of Architecture and Civil Engineering, 1997, 278 pp. (In Russian)

21. Rabotnov Iu. N. Mekhanika deformiruemogo tverdogo tela [Mechanics of deformable solids]. Moscow, Nauka, 1979, 744 pp. (In Russian)

22. Moskalik A. D. Analysis of the stress-strain state of a thick-walled misalignment cylinder under internal pressure in steady-state creep by small parameter method, Proceedings of the Ninth All-Russian Scientific Conference with international participation. Part 1, Matem. Mod. Kraev. Zadachi. Samara, Samara State Technical Univ., 2013, pp. 140-144 (In Russian).

23. Moskalik A. D. The application of perturbation method to problem of misaligned tube in conditions of steady-state creep, Vestn. Samar. Gos. Tekhn. Univ. Ser. Fiz.-Mat. Nauki, 2013, no. 4(33), pp. 76-85 (In Russian). doi: 10.14498/vsgtu1290.

24. Malinin N. N. Prikladnaia teoriia plastichnosti i polzuchesti [Applied Theory of Plasticity and Creep]. Moscow, Mashinostroenie, 1975, 400 pp. (In Russian)

25. Radchenko V. P., Saushkin M. N. Polzuchest' $i$ relaksatsiia ostatochnykh napriazhenii v uprochnennykh konstruktsiiakh [Creep and Relaxation of Residual Stresses in Hardened Structures]. Moscow, Mashinostroenie-1, 2005, 226 pp. (In Russian)

Received 26/V/2014; received in revised form 18/VI/2014; accepted 27/VIII/2014. 